\title{
Effect of Wall Conduction on Natural Convection over a Vertical Slender Hollow Circular Cylinder
}

\author{
TSUNG-YEN NA \\ Department of Mechanical Engineering, School of Engineering, University of Michigan, Dearborn, \\ MI 48128-1491, U.S.A.
}

Received 21 September 1993; accepted in revised form 8 November 1994

\begin{abstract}
An analysis is made of the laminar natural convection of incompressible fluids over a slender, hollow circular cylinder with the inner surface at a constant temperature of $T_{b}$. The temperature of the outer surface must be solved from the coupled conduction of the cylinder and the natural convection of the fluid over the cylinder. The objective of this paper is to investigate the effect of conduction on the heat transfer characteristics of the natural convection boundary layer of the fluid. A wall conduction parameter, $p$, is introduced which is a measure of the heat conductivities of the solid and the fluid and the thickness of the cylindrical shell. The governing differential equations, being non-similar, are solved by a finite-difference method. Numerical results are generated for a series of values of $p$ 's and Prandtl numbers.

The present analysis shows that the overall effect of conduction of the cylinder is to reduce the heat transfer. Any calculation of the heat transfer rate based on the assumption of a constant wall temperature overestimates this quantity. The effect increases for large values of $p$.
\end{abstract}

\section{Nomenclature}

$C_{p}=$ specific heat

$f, g=$ similarity variables

$\bar{g}=$ gravitational acceleration

$\mathrm{Gr}=$ Grashof number

$k=$ heat conductivity

$L \quad=$ length of cylinder

$p=$ axial conduction parameter defined in Eq. (19)

$\operatorname{Pr}=$ Prandtl number

$q=$ heat transfer

$r=$ coordinate in the $r$-direction

$r_{i}, r_{0}=$ inner and outer radii of the hollow cylinder

$R=$ ratio of adjacent interval in $\eta$-direction

$\operatorname{Re}=$ Reynold number

$T_{b}=$ temperature at the inner surface of the hollow cylinder

$T=$ temperature of the fluid

$T_{s}=$ temperature of the cylinder $u_{c}=$ reference velocity

$v_{r}=$ velocity component in $r$-direction

$v_{z}=$ velocity component in $z$-direction

$z \quad=$ coordinate along the axis

Greek symbols

$\rho=$ density

$\nu \quad=$ kinematic viscosity

$\alpha=$ thermal diffusivity

$\beta=$ bulk modulus

$\theta=$ dimensionless temperature

$\psi=$ stream function

\section{Subscripts}

$b \quad=$ condition for $x \leq L$

$\infty=$ condition at infinity in $y$-direction

$f \quad=$ fluid

$s \quad=$ solid 


\section{Introduction}

It is well known that when convective heat transfer rate depends strongly on the thermal boundary condition, it is necessary to consider the problem as a conjugate problem between the solid and the surrounding fluid. An example of such cases is the natural convection over an external surface, as treated in [1-8]. Unlike the classic solutions of natural convections where the thermal boundary condition on the wall is specified in the form of either a specified wall temperature or a specified heat flux, the nonlinear boundary layer equations of the natural convection flow must be solved simultaneously with the energy equation of the conduction heat transfer through the solid. Despite its importance, this class of problems has not received its proper share of attention since the pioneering work of [1] due mainly to the complexity in matching a nonlinear solution of the natural convection boundary layer equations in the fluid with a linear conduction solution in the solid body at the solid-fluid interface. Recently, renewed interest in the conjugate natural convection problems led to some fundamental works on both external [6-8] and internal [9, 10] natural convection problems. For the external conjugate natural convection problems, the emphasis in these works is concentrated on the problem of natural convection over a flat plate where one side is exposed to a given temperature or a given heat flux while heat is transferred to the other side by conduction and then from the solid-liquid interface to the fluid by natural convection. Miyamoto et al. [6] formulated the problem by considering a two-dimensional conduction in the plate and a matching scheme was introduced in which the plate temperature was solved by Fourier series and the natural convection of the fluid by local similarity and the two solutions were matched at the interface. An iterative process was required and the solution was complicated. A major simplification was made recently in which the axial conduction term in the energy equation of the plate was neglected even though the plate temperature was a function of both $x$ - and $y$-directions. Not only the solution process became considerably simplified, the results were found to be very close to the ones using two-dimensional conduction equation of the plate. Using this simplification as a basis, Timma and Padet [7] made an extensive analysis and a family of solutions was generated. Pozzi and Lupo [8] recently made a study of the solution of the equations in [7] by the method of perturbation and the radius of convergence of the series solution was sought.

In this paper, a similar analysis is made of the conjugate problem of the natural convection flow over the outside surface of a slender hollow circular cylinder, as shown in Fig. 1. The temperature at the inner surface of the cylinder is kept at $T_{b}$ and the temperature of the outer surface is determined by the conjugate solution of the energy equation of the solid and the boundary layer equations of the fluid. If the heat conductivity of the hollow cylinder is infinity, the temperature of the cylinder is expected to be equal to $T_{b}$ for the entire hollow cylinder and the solution of the natural convection boundary layer equations based on a constant wall temperature will be applicable. Such is not the case in reality where the heat conductivity of the 


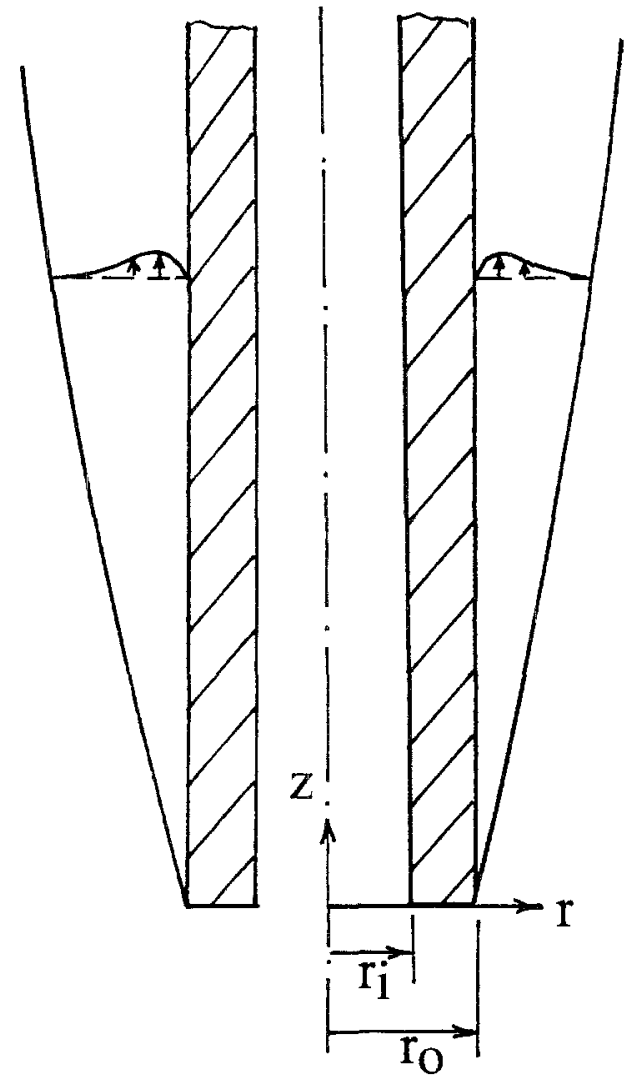

Fig. 1. The hollow cylinder.

plate is finite and a conjugate conduction-convection interaction will therefore be present. It is the purpose of this paper to investigate the effect of heat conduction on the heat transfer rate.

The analysis will follow the lead in [6-8] in that the axial conduction terms in the energy equation of the cylinder is omitted even though the solid temperature is a function of both $r$ and $z$. Analysis based on this assumption can be expected to be very close to the solutions obtained with two-dimensional conduction terms present [7]. The partial differential equations of the fluid in the boundary layer, being non-similar, will be solved by a finite-difference method. Numerical solutions are generated for a set of values of Prandtl numbers and a conduction parameter, $p$.

\section{Analysis}

The governing differential equations for the laminar natural convection flow of an incompressible fluid with constant physical properties except the density and 
under Boussinesq approximation over a vertical circular cylinder (see Fig. 1) can be written as

$$
\begin{aligned}
& \frac{\partial\left(r v_{z}\right)}{\partial z}+\frac{\partial\left(r v_{r}\right)}{\partial r}=0 \\
& v_{z} \frac{\partial v_{z}}{\partial z}+v_{r} \frac{\partial v_{z}}{\partial r}=\frac{\nu}{r} \frac{\partial}{\partial r}\left\{r \frac{\partial v_{z}}{\partial r}\right\}+\bar{g} \beta\left(T-T_{\infty}\right), \\
& v_{z} \frac{\partial T}{\partial z}+v_{r} \frac{\partial T}{\partial r}=\frac{\alpha}{r} \frac{\partial}{\partial r}\left\{r \frac{\partial T}{\partial r}\right\} .
\end{aligned}
$$

Before the boundary conditions can be given, let us make an analysis of the conduction through the cylinder. The two-dimensional heat conduction equation of the hollow circular cylinder can be written as

$$
\frac{1}{r} \frac{\partial}{\partial r}\left(r \frac{\partial T_{s}}{\partial r}\right)+\frac{\partial^{2} T_{s}}{\partial z^{2}}=0
$$

If equation (4) is normalized by introducing the dimensionless quantities

$$
\bar{r}=\frac{r}{r_{0}}, \quad \bar{z}=\frac{z}{L}, \quad \theta_{s}=\frac{T_{s}-T_{\infty}}{T_{b}-T_{\infty}},
$$

equation (4) becomes

$$
\frac{1}{r} \frac{\partial}{\partial \bar{r}}\left(\bar{r} \frac{\partial \theta_{s}}{\partial \bar{r}}\right)+\left(\frac{r_{0}}{L}\right)^{2} \frac{\partial^{2} \theta_{s}}{\partial \bar{z}^{2}}=0 .
$$

Since the ratio $r_{0} / L$ is small, a simplification was introduced $[7,8]$ by omitting the axial conduction term in equation (6). This is a major simplification from the mathematical point of view since it eliminates the iterative process in matching the convective part of the solution. The solution using this simplification was found in general accurate [7]. It should be emphasized that the omission of the axial conduction term does not mean that the temperature of the solid cylinder is independent of $z$. Simplifications of this type are abundant in engineering analysis. A well-known example is the solution of incompressible boundary layer equations over an external body in which the solution of the potential flow is solved over a solid body by assuming no boundary layer. The velocity of slip over the body is then used as the velocity of the mainflow at the edge of the boundary layer.

Under this simplification, the solution of equation (6), subject to the boundary condition: $r=r_{i}: T_{s}=T_{b} ; r=r_{0}: T_{s}=T\left(z, r_{0}\right)$, can be written as

$$
T_{s}=T_{b}+\left\{T\left(z, r_{0}\right)-T_{b}\right\} \frac{\ln r / r_{i}}{\ln r_{0} / r_{i}} .
$$

We therefore get the thermal boundary condition on the outside surface of the cylinder as

$$
r=r_{0}: \quad-k_{s} \frac{\partial T_{s}\left(r_{0}\right)}{\partial r}=-k_{f} \frac{\partial T\left(z, r_{0}\right)}{\partial r} .
$$


Substituting the equation of the temperature of the cylinder, equation (9), into equation (10), we get

$$
y=0: \quad-k_{s} \frac{\left[T\left(z, r_{0}\right)-T_{b}\right]}{r_{0} \ln r_{0} / r_{i}}=-k_{f} \frac{\partial T\left(z, r_{0}\right)}{\partial r},
$$

where $y$ is defined as $y=r-r_{0}$.

The rest of the boundary conditions are

$$
\begin{aligned}
& y=0: \quad v_{z}(z, 0)=0 ; v_{r}(z, 0)=0 ; \\
& y=\infty: v_{z}(z, \infty)=0 ; \quad T(z, \infty)=T_{\infty} .
\end{aligned}
$$

Let us introduce the following dimensionless quantities:

$$
\begin{aligned}
& \bar{z}=\frac{z}{r_{0}} ; \quad \bar{y}=\frac{y}{r_{0}} \sqrt{\operatorname{Re}} ; \quad \bar{v}_{z}=\frac{v_{z}}{u_{c}} ; \quad \bar{v}_{r}=\frac{v_{r}}{u_{c}} \sqrt{\operatorname{Re}} ; \bar{r}=\frac{r}{r_{0}} ; \\
& \theta=\frac{T-T_{\infty}}{T_{b}-T_{\infty}} ; \quad u_{c}=\sqrt{\bar{g} \beta\left(T_{b}-T_{\infty}\right) r_{0}} ; \quad \operatorname{Re}=\frac{u_{c} r_{0}}{\nu}=\sqrt{\mathrm{Gr}} .
\end{aligned}
$$

Equations (1)-(3) become

$$
\begin{aligned}
& \frac{\partial\left(\bar{r} \bar{v}_{z}\right)}{\partial \bar{z}}+\frac{\partial\left(\bar{r} \bar{v}_{r}\right)}{\partial \bar{y}}=0, \\
& \bar{v}_{z} \frac{\partial \bar{v}_{z}}{\partial \bar{z}}+\bar{v}_{r} \frac{\partial \bar{v}_{z}}{\partial \bar{y}}=\frac{1}{\bar{r}} \frac{\partial}{\partial \bar{y}}\left\{\bar{r} \frac{\partial \bar{v}_{z}}{\partial \bar{y}}\right\}+\theta, \\
& \bar{v}_{z} \frac{\partial \theta}{\partial \bar{z}}+\bar{v}_{r} \frac{\partial \theta}{\partial \bar{y}}=\frac{1}{\operatorname{Pr}} \frac{1}{\bar{r}} \frac{\partial}{\partial \bar{y}}\left\{\bar{r} \frac{\partial \theta}{\partial \bar{y}}\right\},
\end{aligned}
$$

subject to the boundary conditions

$$
\begin{aligned}
& \bar{y}=0: \quad \bar{v}_{z}(\bar{z}, 0)=0 ; \quad \bar{v}_{r}(\bar{z}, 0)=0 ; \\
& \theta(\bar{z}, 0)-1=p \frac{\partial \theta(\bar{z}, 0)}{\partial \bar{y}} ; \\
& \bar{y}=\infty: \quad \bar{v}_{z}(\bar{z}, \infty)=0 ; \quad \theta(\bar{z}, \infty)=0,
\end{aligned}
$$

where

$$
p=\frac{k_{f}}{k_{s}} \ln \frac{r_{0}}{r_{i}} \mathrm{Gr}^{1 / 4} .
$$

Inspection of equations (13-18) shows that the solution of this problem now depends on two dimensionless parameters, namely, the Prandtl number Pr and the conduction parameter $p$, respectively. In the limit when $p$ becomes zero, the thermal 
boundary condition on the wall becomes that of a constant wall temperature. The parameter $p$ therefore is a measure of deviation from the constant wall temperature solution. From the definition of the conduction parameter, $p$, it is seen that a constant wall temperature solution is accurate only if the ratio of the heat conductivity of the fluid to that of the solid is small and/or the thickness of the tube is thin. Another point that needs to be emphasized is that for this problem, the Biot number is no longer adequate since it does not include all the physical parameters involved in this problem.

Let us next introduce the Mangler Transformation

$$
d z^{*}=d \bar{z} ; \quad d y^{*}=\bar{r} d \bar{y}
$$

and the stream function, $\bar{\psi}$, defined by

$$
\bar{r} \bar{v}_{z}=\frac{\partial \bar{\psi}}{\partial \bar{y}} ; \quad \bar{r} \bar{v}_{r}=-\frac{\partial \bar{\psi}}{\partial \bar{z}} .
$$

Equation (13) is satisfied identically and equation (14) becomes

$$
\frac{\partial \bar{\psi}}{\partial y^{*}} \frac{\partial^{2} \bar{\psi}}{\partial z^{*} \partial y^{*}}-\frac{\partial \bar{\psi} \partial^{2} \bar{\psi}}{\partial z^{*} \partial y^{* 2}}=\frac{\partial}{\partial y^{*}}\left\{\left(1+\frac{2 y^{*}}{\sqrt{\mathrm{Re}}}\right) \frac{\partial^{2} \bar{\psi}}{\partial y^{* 2}}\right\}+\theta
$$

In a similar manner, equation (15) can be reduced to

$$
\frac{\partial \bar{\psi}}{\partial y^{*}} \frac{\partial \theta}{\partial z^{*}}-\frac{\partial \bar{\psi}}{\partial z^{*}} \frac{\partial \theta}{\partial y^{*}}=\frac{1}{\operatorname{Pr}} \frac{\partial}{\partial y^{*}}\left\{\left(1+\frac{2 y^{*}}{\sqrt{\operatorname{Re}}}\right) \frac{\partial \theta}{\partial y^{*}}\right\}
$$

subject to the boundary conditions

$$
\begin{aligned}
& y^{*}=0: \quad \frac{\partial \bar{\psi}\left(z^{*}, 0\right)}{\partial y^{*}}=0 ; \quad \frac{\partial \bar{\psi}\left(z^{*}, 0\right)}{\partial z^{*}}=0 ; \\
& \theta\left(z^{*}, 0\right)-1=p \frac{\partial \theta\left(z^{*}, 0\right)}{\partial y^{*}} ; \\
& y^{*}=\infty: \quad \frac{\partial \bar{\psi}\left(z^{*}, \infty\right)}{\partial y^{*}}=0 ; \quad \theta\left(z^{*}, \infty\right)=0 .
\end{aligned}
$$

Finally, let us introduce the following transformation:

$$
\begin{aligned}
\zeta & =\frac{2 z^{*(1 / 5)}}{\sqrt{\operatorname{Re}}} ; \quad \eta=\frac{y^{*}}{z^{*(1 / 5)}} ; \quad f(\zeta, \eta)=\frac{\bar{\psi}}{z^{*(4 / 5)}} ; \\
g(\zeta, \eta) & =\frac{\theta}{z^{*(1 / 5)}} .
\end{aligned}
$$

Equations (22) and (23) and the boundary conditions, equations (24)-(26), become

$$
\left\{(1+\zeta \eta) f^{\prime \prime}\right\}^{\prime}+\frac{4}{5} f f^{\prime \prime}-\frac{3}{5}\left\{f^{\prime}\right\}^{2}+g=\frac{\zeta}{5}\left\{f^{\prime} \frac{\partial f^{\prime}}{\partial \zeta}-f^{\prime \prime} \frac{\partial f}{\partial \zeta}\right\}
$$




$$
\frac{1}{\operatorname{Pr}}\left\{(1+\zeta \eta) g^{\prime}\right\}^{\prime}+\frac{4}{5} f g^{\prime}-\frac{1}{5} f^{\prime} g=\frac{\zeta}{5}\left\{f^{\prime} \frac{\partial g}{\partial \zeta}-g^{\prime} \frac{\partial f}{\partial \zeta}\right\}
$$

subject to the boundary condition

$$
\begin{aligned}
& \eta=0: \quad f(\zeta, 0)=0 ; \quad f^{\prime}(\zeta, 0)=0 ; \\
& \frac{\sqrt{\operatorname{Re}}}{2} \zeta g(\zeta, 0)-1=p g^{\prime}(\zeta, 0) ; \\
& \eta=\infty: \quad f^{\prime}(\zeta, \infty)=0 ; \quad g(\zeta, \infty)=0 .
\end{aligned}
$$

\section{Solutions and Results}

To solve the system of partial differential equations, equations (28) and (29), subject to the boundary conditions, equations (30)-(32), the finite-difference method is used. The system of partial differential equations are first written as a first-order system. The derivatives are then approximated by centered-difference gradients and averages centered at the mid-points of the net rectangles defined by

$$
\begin{aligned}
& \zeta_{0}=0, \quad \zeta_{n}=\zeta_{n-1}+k_{n} ; \quad n=1,2, \ldots, N \\
& \eta_{0}=0, \quad \eta_{j}=\eta_{j-1}+h_{j}, \quad j=1,2, \ldots, J \\
& \eta_{J}=\eta_{\infty} .
\end{aligned}
$$

A non-uniform grid $h_{j}$ defined by

$$
h_{j}=R h_{j-1},
$$

where the ratio of adjacent intervals, $R$, is a constant. The distance from the surface to the $j$-th station is then given by

$$
\eta_{j}=h_{j} \frac{R^{j}-1}{R-1}, \quad j=1,2, \ldots, J .
$$

The $\zeta$-direction grid $k_{n}$ is arbitrary. Linearization is achieved by the method of quasilinearization and the resulting system of algebraic equations are then solved by a block-tridiagonal factorization technique described, e.g., in [11]. Details of the method are identical to the one used in [11] and are therefore omitted here.

Numerical solutions are generated using the above scheme for three values of Prandtl numbers $(0.72,5$ and 20$)$ and for three values of the wall conduction parameter, $p(0.1,0.2$ and 0.3$)$. A variable grid with $h_{0}$ and $R$ chosen to be 0.01 and 1.01, respectively. The ratio $L / r_{0}$ is selected to be 20 for all the numerical solutions. The solutions presented are for a Grashof number of $1.25 \times 10^{5}$. At these values of $\mathrm{Gr}$ and $L / r_{0}$, the flow remains laminar for the entire length of the cylinder. 


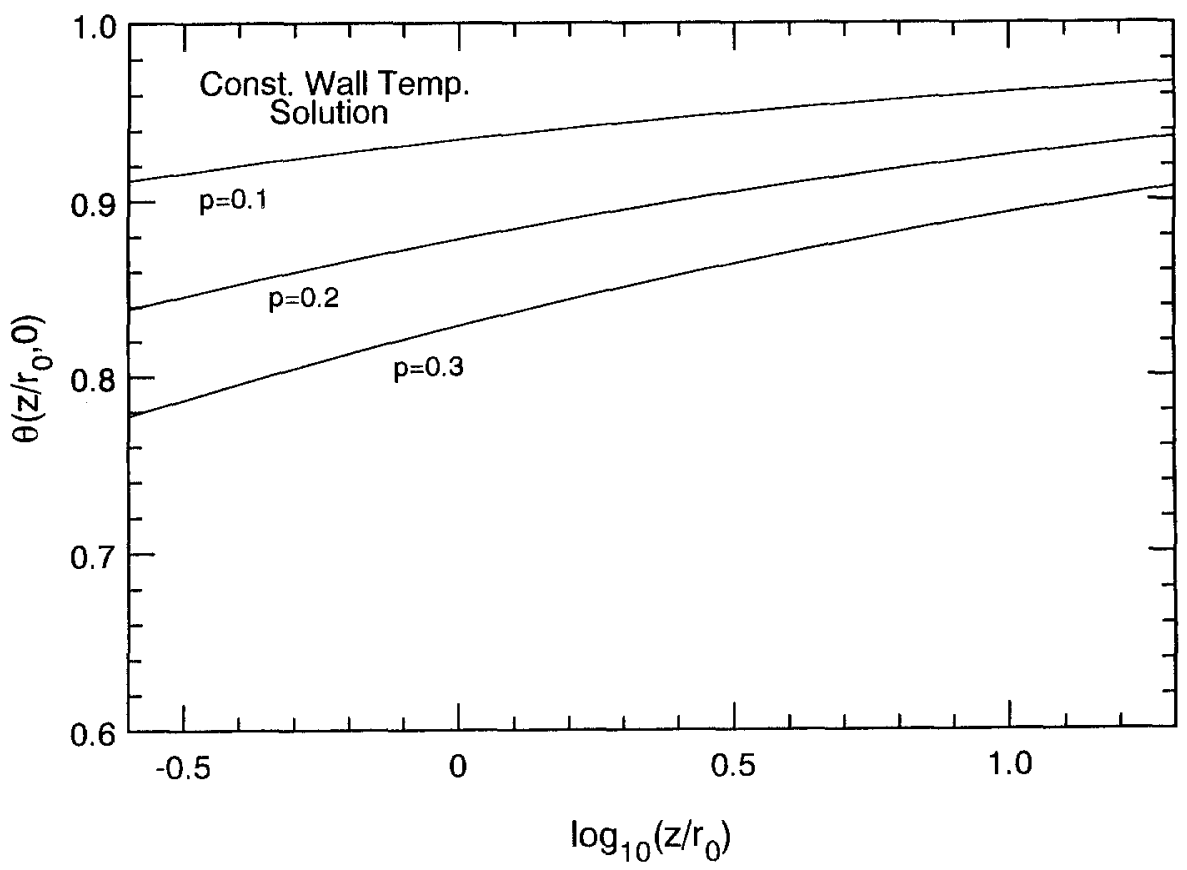

Fig. 2. $\theta\left(z / r_{0}, 0\right)$ versus $z / r_{0}(\operatorname{Pr}=5)$.

Let us first consider the dimensionless temperature of the fluid on the surface of the cylinder which, in terms of $\zeta$ and $g(\zeta, 0)$, can be written as

$$
\theta(\bar{z}, 0)=\frac{\mathrm{Gr}^{1 / 4}}{2} \zeta g(\zeta, 0)
$$

where the coordination along the axis of the cylinder, $\zeta$ is related to $\bar{z}$ by the equation

$$
\bar{z}=\frac{z}{r_{0}}=\left\{\frac{\zeta \mathrm{Gr}^{1 / 4}}{2}\right\}^{5} .
$$

For a hollow cylinder where the temperature on the inner surface $\left(r=r_{i}\right)$ is maintained at a constant value of $T_{b}$, the temperature on the outer surface of the cylinder $\left(r=r_{0}\right)$ depends on the coupled solution of the conduction through and the natural convection of the fluid over the circular cylinder. Intuitively, if the heat conductivity of the hollow cylinder is very large, its temperature can be expected to be approximately uniform at $T_{b}$ and therefore a solution of the natural convection based on a constant wall temperature will be acceptable. An inspection of the definition of the conduction parameter $p$ reveals that the same conclusion is true if the heat conductivity of the fluid is very small and/or if the thickness of the hollow cylinder is very thin.

Figure 2 shows the dimensionless wall temperature of the fluid, $\theta\left(z / r_{0}, 0\right)$, as a function of the coordinate along the axis of the cylinder, i.e., $\bar{z}$, for a Prandtl number 


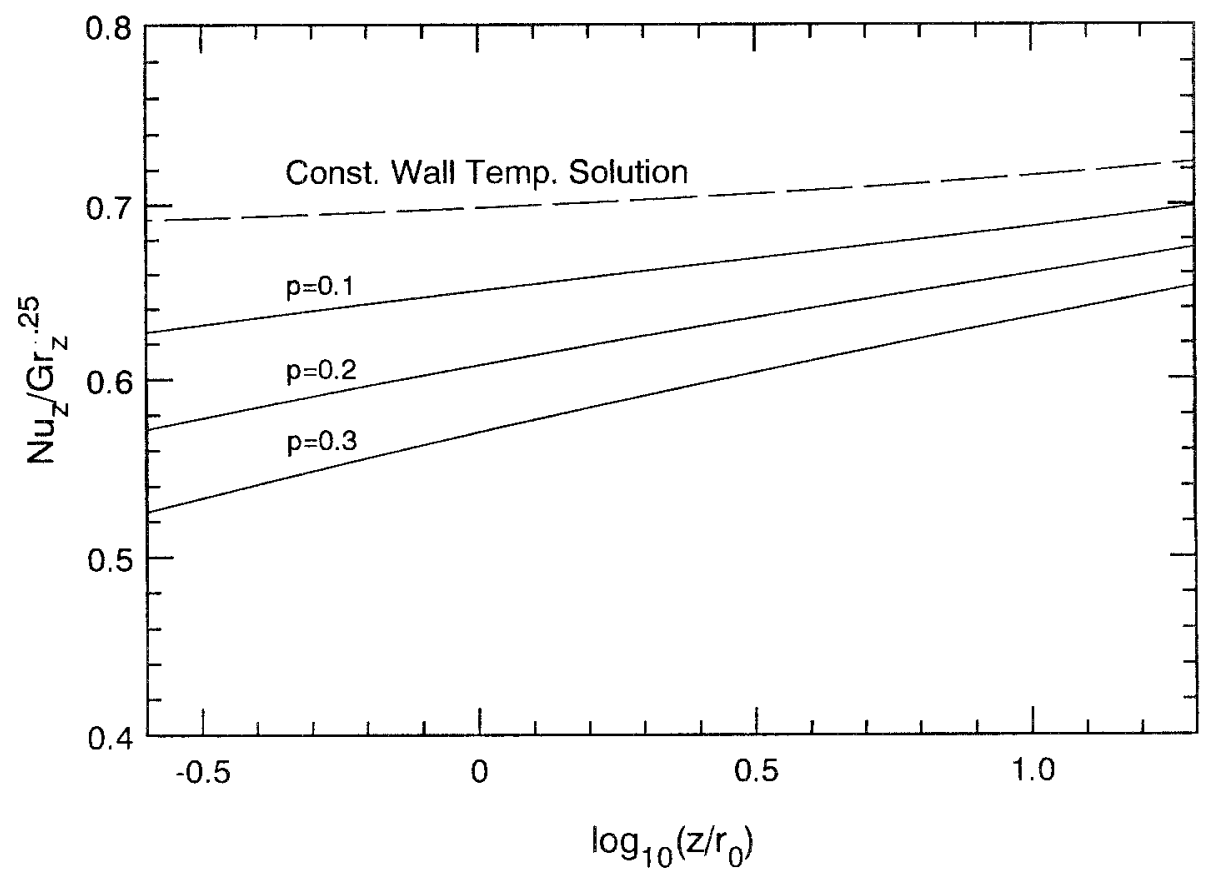

Fig. 3. $\mathrm{Nu}_{z} / \mathrm{Gr}_{z}^{0.25}$ versus $z / r_{0}(\mathrm{Pr}=5)$.

of 5 and three values of the conduction parameter, $p$. To demonstrate the effect of wall conduction, it is necessary to point out that the solution of the basic case $(p=0)$ where the wall temperature is assumed to be uniform at $T_{b}$ is represented by a horizontal straight line in Fig. 2 since for this case

$$
\theta\left(z / r_{0}, 0\right)=1
$$

for all $z$ 's.

The deviation of the temperature of the fluid on the surface from the straight line, equation (38), as shown in Fig. 2, therefore represents the effect of conduction. The behavior of the solution depends on two dimensionless parameters, namely, $p$ and Pr. Figure 2 shows that the effect of $p$ on the temperature of the outer surface of the cylinder for a given Prandtl number to be a decrease in the outer surface temperature from the constant value in equation (38) in the basic case. The surface temperature is lowest at the leading edge and increase along the outer surface until it approaches to the constant value of the basic case at very large $z$.

Let us next consider the effect of conduction on the rate of heat transfer. First, the local rate of heat transfer is given by

$$
\dot{q}_{w}(z)=-k_{f} \frac{\partial T(z, 0)}{\partial y}=k_{f} \frac{T_{b}-T_{\infty}}{r_{0}} \mathrm{Gr}^{1 / 4}\left[-g^{\prime}(\zeta, 0)\right] .
$$

Let us define the overall local heat transfer coefficient, $h_{z}$, as

$$
\dot{q}_{w}(z)=h_{z}\left(T_{b}-T_{\infty}\right)
$$




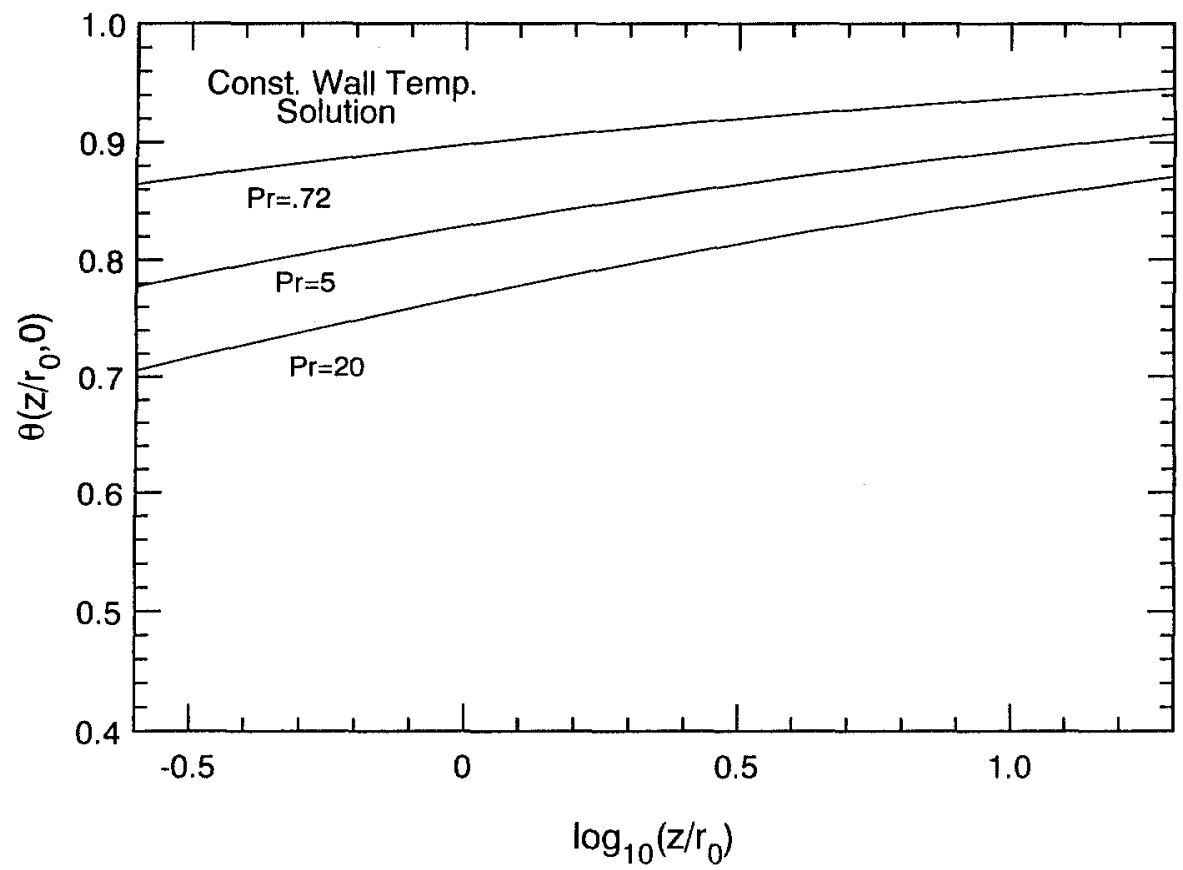

Fig. 4. $\theta\left(z / r_{0}, 0\right)$ versus $z / r_{0}(p=0.3)$.

and the local Nusselt number is defined as

$$
\mathrm{Nu}_{z}=\frac{h_{z} z}{k_{f}} ;
$$

we therefore get

$$
\frac{\mathrm{Nu}_{z}}{\mathrm{Gr}_{z}^{1 / 4}}=\bar{z}^{1 / 4}\left[-g^{\prime}(\zeta, 0)\right]
$$

where

$$
\mathrm{Gr}_{z}=\frac{\bar{g} \beta\left(T_{b}-T_{\infty}\right) z^{3}}{\nu^{2}} .
$$

Figure 3 is a plot of equation (39) for $\operatorname{Pr}=5$ and three values of $p$, namely, $0.1,0.2$ and 0.3 , respectively. The basic solution of constant wall temperature case $(p=0)$ is also plotted in Fig. 3 as a dotted line. The general trend is a decreases of local Nusselt number as compared with the basic solution of constant wall temperature case. The decrease is seen increase for larger values of $p$.

Figures 4 and 5 show the effect of Prandtl number. The value of the parameter $p$ is taken to be a constant at 0.3 with three Prandtl numbers, namely, 0.72, 5 and 20, respectively. Figure 4 shows that for a given $p$, the effect of heat conduction of the temperature of the fluid on the outer surface of the cylinder is more significant for larger values of $\operatorname{Pr}$, as compared with the basic solution $(p=0)$ of the constant wall 


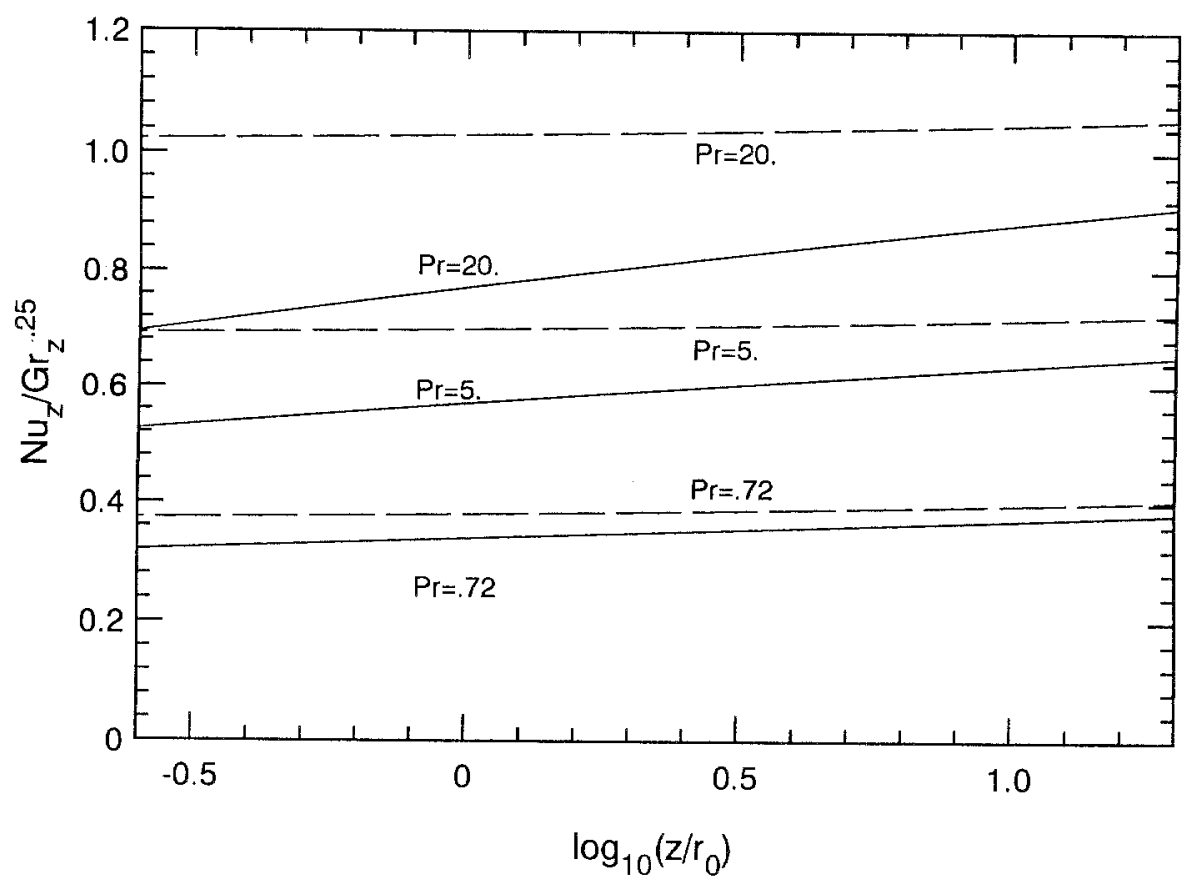

Fig. 5. $\mathrm{Nu}_{z} / \mathrm{Gr}_{z}^{0.25}$ versus $z / r_{0}(p=0.3)$.

TABLE I. Representative solutions $(\operatorname{Pr}=5)$

\begin{tabular}{rlll}
\hline$z / r_{0}$ & $p=0.1$ & $p=0.2$ & $p=0.3$ \\
\hline 0.043 & 0.5907 & 0.5175 & 0.4601 \\
0.111 & 0.6104 & 0.5471 & 0.4952 \\
0.271 & 0.6280 & 0.5739 & 0.5279 \\
0.678 & 0.6439 & 0.5981 & 0.5580 \\
1.694 & 0.6588 & 0.6203 & 0.5857 \\
4.230 & 0.6734 & 0.6411 & 0.6115 \\
10.555 & 0.6881 & 0.6611 & 0.6359 \\
\hline
\end{tabular}

temperature case. Lower surface temperatures therefore result in lower Nusselt numbers, as shown in Fig. 5. Again, the basic solutions of the constant wall temperature case for each Prandtl number are plotted as dotted lines. The difference between solid and dotted lines for each Prandtl number is larger for larger values of Prandtl numbers which means the effect of conduction is more pronounced for larger Prandtl numbers.

Table 1 gives some representative solutions of the problem for future reference. 


\section{Conclusions}

An analysis is made of the natural convection over a slender, hollow circular cylinder with the inner surface at a constant temperature $T_{b}$ but the temperature of the outer surface must be solved from the coupled conduction of the cylinder and the natural convection of the fluid over the cylinder. The objective is to investigate the effect of conduction on the heat transfer of the natural convection boundary layer of the fluid. A wall conduction parameter, $p$, is identified which is a measure of the heat conductivities of the solid and the fluid and the thickness of the cylindrical shell, as defined by equation (19).

The present analysis shows that the overall effect of heat conduction is to reduce the rate of heat transfer. Any calculation of the heat transfer rate based on the assumption of a constant wall temperature of $T_{b}$ over the entire surface of the cylinder overestimates this quantity. Consider, e.g., the problem of natural convection of water over a hollow steel cylinder with $r_{i}=0.025 \mathrm{~m}$ and $r_{e}=$ $0.035 \mathrm{~m}$. The value of $p$ then equals to 0.1 . From the present analysis, the effect of conduction cannot be neglected. On the other hand, if the solid cylinder is made of aluminium, the conduction parameter becomes 0.02 . For such a small value of $p$, neglecting the wall heat conduction becomes an acceptable assumption. The magnitude of the decrease depends on the value of the parameter $p$ and the Prandtl number. The effect increases for larger values of $p$ and/or Pr.

\section{References}

1. Kelleher, M.D. and Yang, K.T., A steady conjugate heat transfer problem with conduction and free convection. Appl. Sci. Res. 17 (1967) 240-268.

2. Lock, G.S.H. and Gunn, J.C., Laminar free convection from a downward projecting fin. J. Heat Transfer (1968) 63-70.

3. Ziness, A.E., The coupling of conduction with laminar natural convection from a vertical flat plate with arbitrary surface heating. J. Heat Transfer 92 (1970) 528-535.

4. Rotem, Z., Conjugate free convection from horizontal conducting circular cylinders. Int. J. Heat Mass Transfer 15 (1972) 1679-1693.

5. Chida, K. and Katto, Y., Study on conjugate heat transfer by vectorial dimensional analysis. Int. J. Heat Mass Transfer 10 (1976) 453-460.

6. Miyamoto, M., Sumikawa, J. and Nakamura, T., Effects of axial heat conduction in a vertical flat plate on free convection heat transfer. Int. J. Heat Mass Transfer 23 (1980) 1545-1553.

7. Timma, J. and Padet, J.P., Etude théorique du couplage convection-conduction en convection libre laminaire sur une plaque verticale. Int. J. Heat Mass Transfer 28 (1985) 1097-1104.

8. Pozzi, A. and Lupo, M., The coupling of conduction with laminar natural convection along a flat plate. Int. J. Heat Mass Transfer 31 (1988) 1807-1814.

9. Kim, S.H., Anand, N.K. and Wing Aung, Effect of wall conduction on free convection between asymmetrically heated vertical plates: uniform wall heat flux. Int. J. Heat Mass Transfer 33 (1990) 1013-1023.

10. Anand, N.K., Kim, S.H. and Wing Aung, Effect of wall conduction on free convection between asymmetrically heated vertical plates: uniform wall temperature. Int. J. Heat Mass Transfer 33 (1990) 1025-1028.

11. Na, T.Y., Computational Methods in Engineering Boundary Value Problems. Academic Press, New York (1979). 